Escape Depth for Excited Photoelectrons in $\mathrm{KBr}$ Films*

\title{
William Pong
}

Department of Physics and Astronomy University of Hawaii, Honolulu, Hawaii

\section{Abstract}

Quantum yields for backward and forward photoemission from evaporated $\mathrm{KBr}$ films have been measured as a function of film thickness. From the results of photoelectric yield measurements, the attenuation length for photoelectrons excited at $\mathrm{hv}=10.2 \mathrm{eV}$ was found to be approximately $180 \AA$, which is slightly greater than the absorption length for the photons. The measured attenuation length was used to estimate the mean free path for electron-phonon collisions.
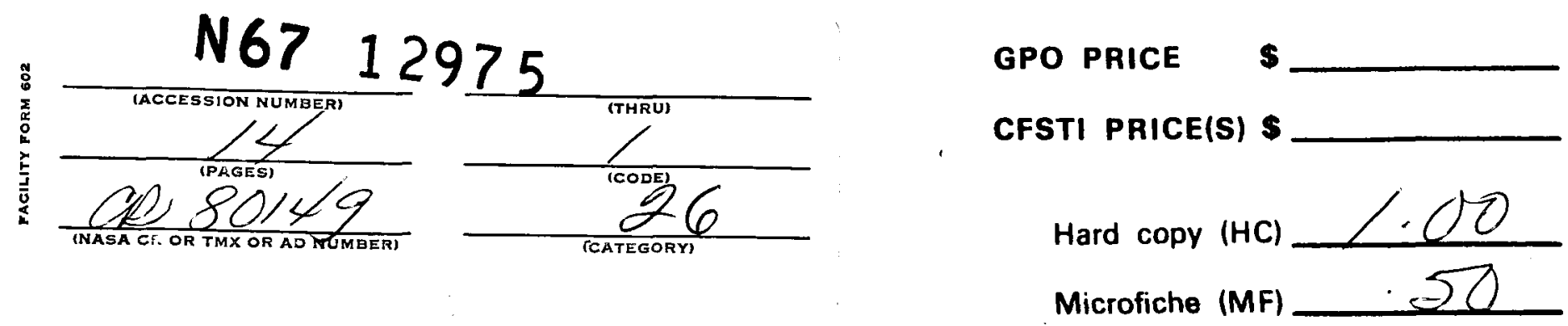

f1 653 July 65

* Supported by NASA Grant NsG-328 


\section{Introduction}

Recent measurements ${ }^{l}$ of photoemission from evaporated alkali halide films in the vacuum ultraviolet have shown that the photoelectric yields of some alkali halides are more than 0.5 at some photon energies above $9 \mathrm{eV}$. The relatively high yield implies that the escape depth for photoexcited electrons in these photoemitters is larger than the absorption length for the photons. In order to obtain further evidence that this is the case, the range of the excited electrons must be investigated. The purpose of the present work has been to determine the attenuation length of the excited photoelectrons in evaporated $\mathrm{KBr}$ films by measuring the quantum yield as a function of film thickness. It is shown that the results of the experiments are useful in estimating the mean free path for electron-phonon collisions in $\mathrm{KBr}$.

\section{Attenuation Length}

The escape probability ${ }^{2}$ of an electron excited at a distance $\mathrm{x}$ from the surface of a solid-vacuum barrier is given by

$$
P(x)=p_{0} e^{-x / L},
$$

where $p_{O}$ is a constant for a given hv and $L$ is the attenuation length. It should be noted that $\mathrm{L}$ depends on the mean free paths for inelastic and elastic collisions. A method of relating $L$ to various values of mean free paths has been discussed by stuart, Wooten, and Spicer ${ }^{3}$.

In using the exponential probability expression to calculate the photoelectric yield, we assume that the incident photons of 
energy hv produce electrons of average kinetic energy $\mathrm{E}$. The value of E may vary with hu. Now consider a photoemissive film of thickness $\mathrm{T}$ as shown in Fig. 1. The quantum yield in number of emitted electrons per absorbed photon can be represented by

$$
Y=p_{0} \int_{0}^{T} \alpha e^{-\alpha S} e^{-(T-S) / L} d s / \int_{0}^{T} \alpha e^{-\alpha S} d s,
$$

where $\alpha$ is the absorption coefficient of the photoemissive layer. Upon evaluating the integrals in Eq. (2), we find

$$
\mathrm{Y}=\alpha \mathrm{Lp} \mathrm{O}_{\mathrm{O}}\left(\mathrm{e}^{-\alpha \mathrm{T}}-\mathrm{e}^{-\mathrm{T} / \mathrm{L}}\right) \cdot /(1-\alpha \mathrm{L})\left(1-\mathrm{e}^{-\alpha \mathrm{T}}\right)
$$

It should be noted that optical reflection and electron scattering at the boundaries were not considered in the derivation of the yield equation.

\section{Experimental Method}

Quantum-yield measurements were made on $\mathrm{KBr}$ films evaporated in situ in a vacuum of $10^{-7}$ Torr. The incident radiation of a particular wavelength was obtained by means of a one-meter normal incidence vacuum monochromator. Fig. 2 illustrates the experimental arrangement. The sample chamber is shown in Fig. 3. It is similar to the apparatus ${ }^{4}$ previously described; the only difference is that the substrate holder and collector are mounted on ' a rotatable feedthrough for measurements of forward emission as well as backward emission. Backward photoemission refers to the situation illustrated in Fig. 1.

Electrical connection between the evaporated layer and the 
electrometer lead was made by a circular strip of silver paint on a thin LiF substrate. The LiF crystal was initially coated with a thin film of gold to ensure adequate electrical contact with the $\mathrm{KBr}$ film, which was evaporated over the gold film. The backward photoemission current from the gold film was small in comparison with that from the $\mathrm{KBr}$ film at $10.2 \mathrm{eV}$.

The thickness of the evaporated film was determined by transmission measurements at $10.2 \mathrm{eV}$. The value ${ }^{5}$ of $\alpha$ for $\mathrm{KBr}$ at $10.2 \mathrm{eV}$ is about $6 \times 10^{5} \mathrm{~cm}^{-1}$. Absorption measurements were made in order to see how the spectral absorption of the evaporated film compare with the reported data on $\mathrm{KBr}$. The results shown in Fig. 4 are in agreement with those of Philipp and Ehrenreich ${ }^{5}$.

\section{Results and Discussion}

As shown in Fig. 5, the yield for backward photoemission from $\mathrm{KBr}$ at hv $=10.2 \mathrm{eV}$ decreases with increasing thickness. For $\mathrm{p}_{\mathrm{O}}=0.34$ and $\mathrm{L}=180 \AA$, the calculated curve from $\mathrm{Eq}$. (3) fits the yield data for thicknesses greater than $150 \AA$. The data obtained at thicknesses greater than $100 \AA$ are probably more reliable for comparison with $\mathrm{Eq}$. (3). The lack of agreement at small thicknesses may be attributed to photoemission from the substrate and other boundary effects which we have neglected in deriving $\mathrm{Eq}$. (3). It is interesting to note that the attenuation length $\mathrm{L}$ is slightly greater than the absorption length for the photons in this case. 
Inasmuch as $\mathrm{L}$ has been determined experimentally, it is worthwhile to estimate the mean free path of the excited electrons in $\mathrm{KBr}$. In this case the mean free path for electron-electron collisions is probably large in comparison with that for electronphonon interaction. Therefore we will assume that the electrons excited at $10.2 \mathrm{eV}$ lose energy only by electron-phonon collisions. For $\mathrm{hv}=10.2 \mathrm{eV}$ and a photoelectric threshold of $7.8 \mathrm{eV}$, the average kinetic energy of the excited photoelectrons is probably $1.2 \mathrm{eV}$. Using the relationship $\mathrm{E}=3 \mathrm{kT} / \mathrm{e} / 2$, we find that $\mathrm{kT}_{\mathrm{e}}$ is about $0.8 \mathrm{eV}$. Since the phonon energy $\mathrm{E}_{\mathrm{r}}$ for $\mathrm{KBr}$ is approximately $0.015 \mathrm{eV}$, we have the condition $\mathrm{kT}_{\mathrm{e}} \gg \mathrm{E}_{\mathrm{r}}$. Consequently, we may consider the photoelectrons as hot electrons. According to theory ${ }^{6}$, a. relationship between the mean free path $\ell$ and the attenuation length $L$ is given by

$$
\ell^{2} \approx 3 \mathrm{E}_{\mathrm{r}} \mathrm{L}^{2} / \mathrm{KT}_{\mathrm{e}}
$$

for $k T_{e} \gg E_{\Gamma}$. Thus for $L=180 \AA$ the mean free path is approximately $42 \stackrel{\circ}{\AA}$.

From a knowledge of the mean free path we may estimate the collision frequency $f$ from the relation

$$
\mathrm{f}=\mathrm{u} / \mathrm{l},
$$

where $u$ is the velocity which can be computed from $E=\mathrm{m} \mathrm{u}^{2} / 2$. For $\mathrm{E}=1.2 \mathrm{eV}$ the electron velocity is about $6.5 \times 10^{7} \mathrm{~cm} / \mathrm{sec}$. Using the values we have for $l$ and $u$, we obtain a frequency of about $1.6 \times 10^{14} \mathrm{sec}^{-1}$, which is in agreement with the estimate given by Seitz ${ }^{7}$.

In addition to the backward photoemission measurements, the 
forward emission was investigated as a function of thickness. The results are shown in Fig. 6. The yield curves appear to saturate at about $200 \AA$. However, a gradual increase in yield is noticeable at larger thicknesses, especially for higher photon energies. The saturation effect is in agreement with the calculated yield for forward photoemission. It can be shown that the quantum yield in number of emitted electrons per incident photon is given by

$$
Y_{i}=\alpha \operatorname{Lp}\left(1-e^{-(\alpha+1 / L) T}\right) /(\alpha L+1)
$$

For large values of $\mathrm{T}$, the yield $\mathrm{Y}_{i}$ is represented by

$$
Y_{i}=\alpha L p_{O} /(\alpha I+1) \text {. }
$$

If we use the values from the backward-emission measurements for po and $\mathrm{L}$ at $10.2 \mathrm{eV}, \mathrm{Eq}$. (7) gives a limiting yield of about 0.18, which is in agreement with the experimental data for thicknesses of about $200 \stackrel{\circ}{\AA}$.

No attempt has been made to explain the additional rise in yield at thicknesses greater than $300 \AA$. One might suspect that the increase in yield is due to surface charges. Since $\mathrm{KBr}$ is an insulator, there is a possibility of charging the photoemissive surface when the film is sufficiently thick. It is well known that high electric field intensity within the film may introduce enhanced emission by producing impact ionization or field , , emission.

The quantum yield for forward photoemission from a $\mathrm{KBr}$ film of $630 \AA$ thick is higher than the yield reported by Taft and Philipp ${ }^{8}$. The discrepancy may be due to different film thickness. 
Footnotes and References

* Supported by NASA Grant NsG-328.

1. Duckett S. W. and Metzger P. H., Phys. Rev. 137, A953 (1965);

Metzger P. H., J. Phys. Chem. Solids, 26, 1879 (1965).

2. Spicer W. E., Phys. Rev. 112, 114 (1958).

3. Stuart R., Wooten F. and Spicer W. E., Phys. Rev. 135, A495 (1964).

4. Pong. W., J. Appl. Phys. 37, 3033 (1966).

5. Philipp H. R. and Ehrenreich H., Phys. Rev. 131, 2016 (1963).

6. Baraff G. A., Phys. Rev. 135., A528 (1964).

7. Seitz F., Phys. Rev. 76, 1376 (1949).

8. Taft E. A. and Philipp H. R., J. Phys. Chem. Solids 3, I (1957). 


\section{Captions}

Fig. I Backward photoemission from a photoemissive film of thickness T. Photoelectrons are produced by radiation which passed through the LiF substrate.

Fig. 2 A sketch of the experimental arrangement: vacuum uv monochromator, sample chamber, and ion-pump system.

Fig. 3 Spectral absorption of evaporated $\mathrm{KBr}$ film. The absorption coefficient at $10.2 \mathrm{eV}$ was assumed to be $6 \times 10^{5}$ $\mathrm{cm}^{-1}$ and the values at other photon energies were calculated from optical-density data.

Fig. 5 Backward photoemission yield of evaporated $\mathrm{KBr}$ film at $10.2 \mathrm{eV}$. The yield $\mathrm{Y}$ in number of emitted electrons per absorbed photon decreases with increasing thickness.

Fig. 6 Forward photoemission yield of evaporated $\mathrm{KBr}$ film at different photon energies. The yield $Y_{i}$ in number of emitted electrons per incident photon increases with increasing thickness. 


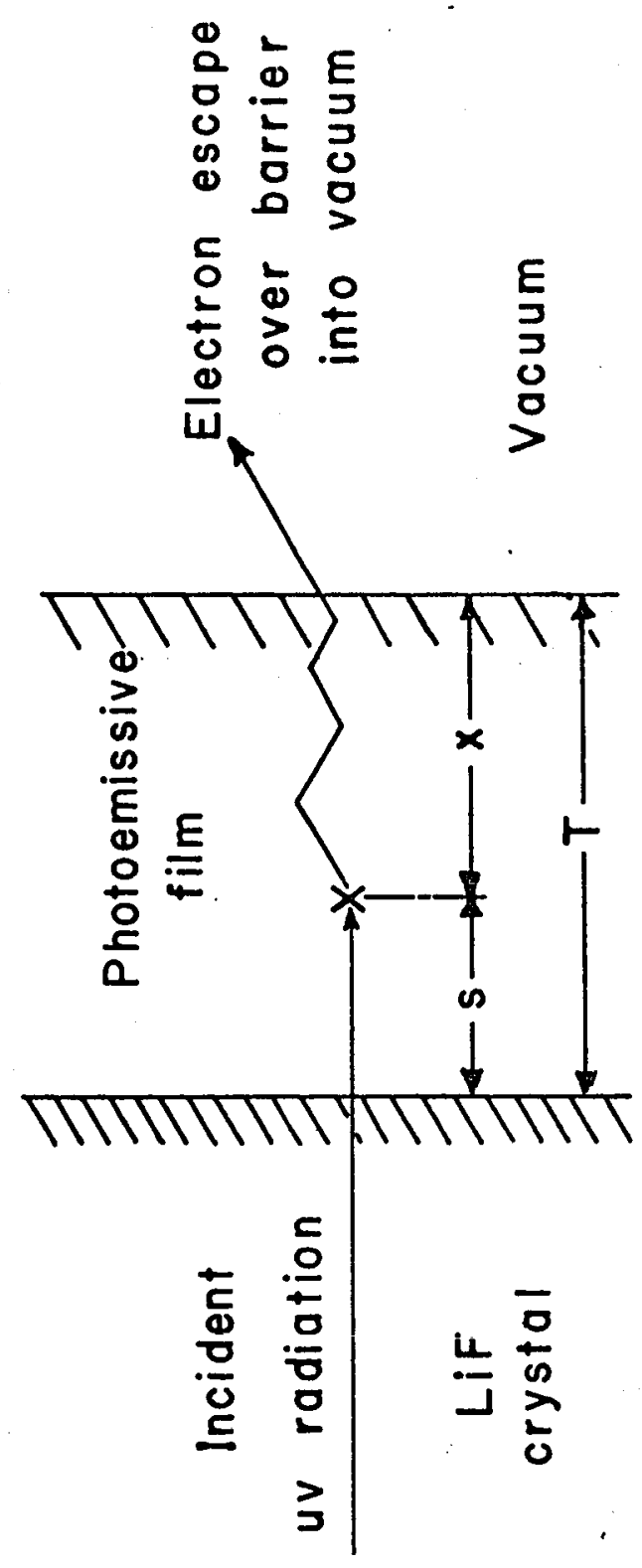


$?$.

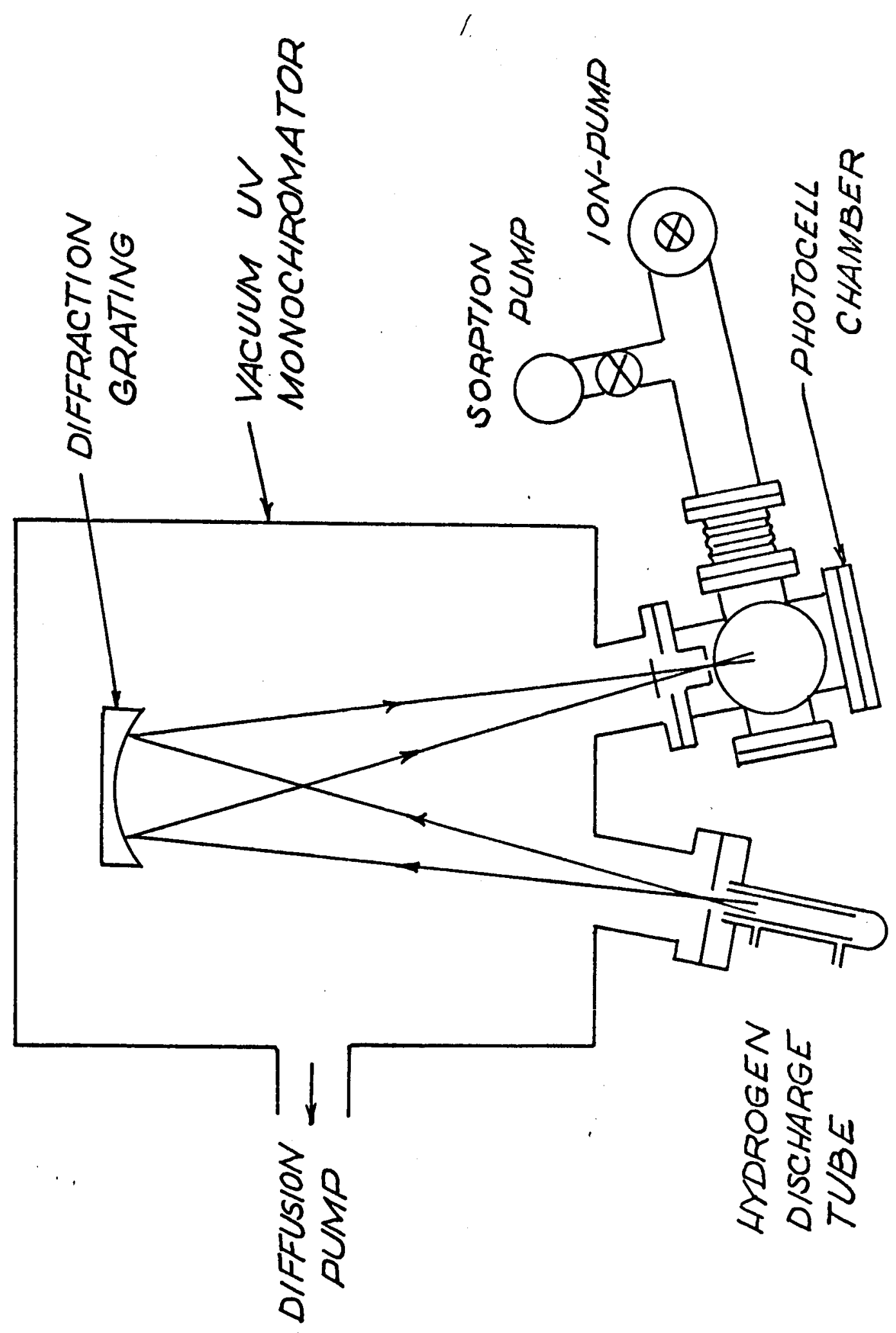




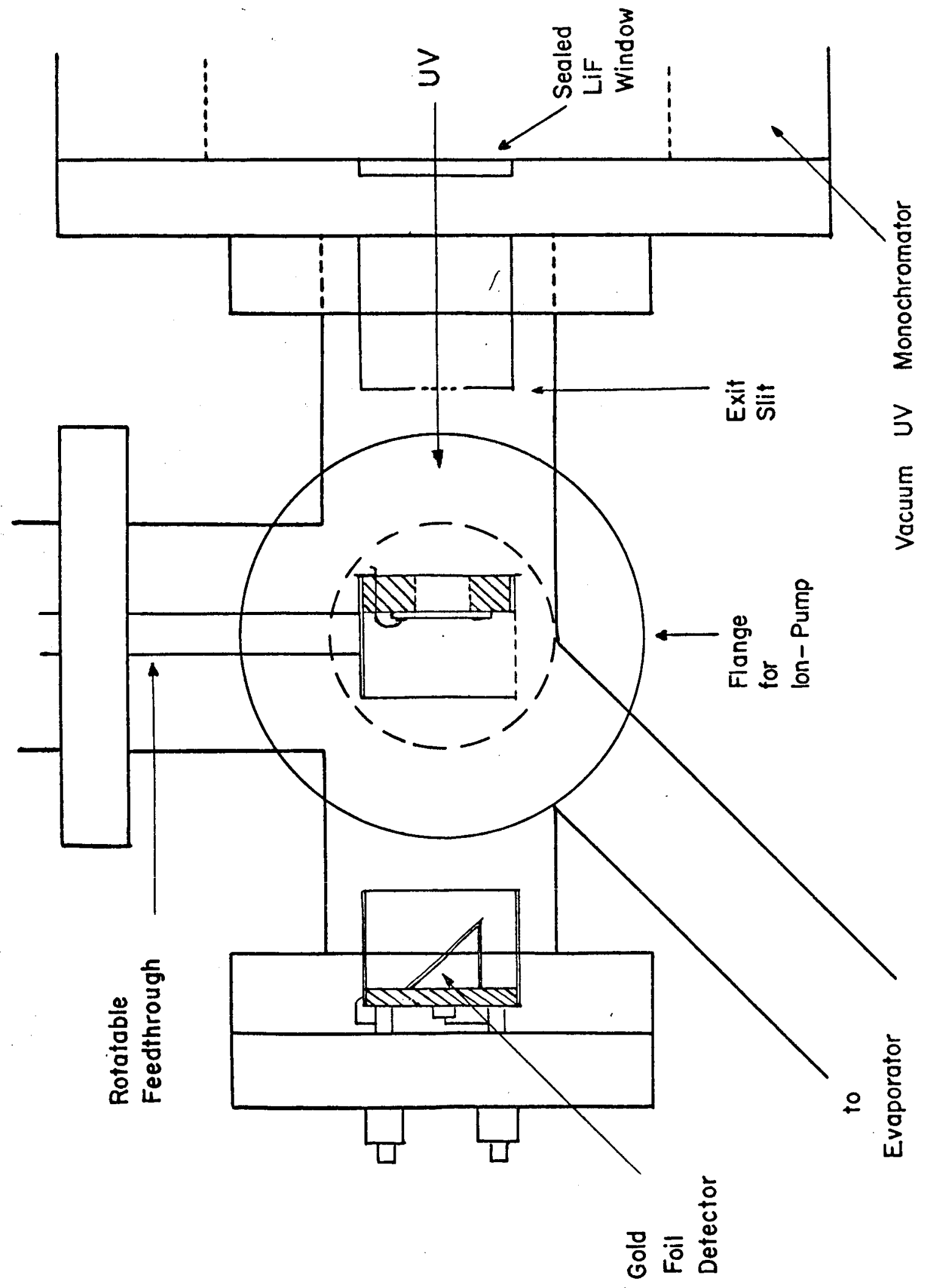




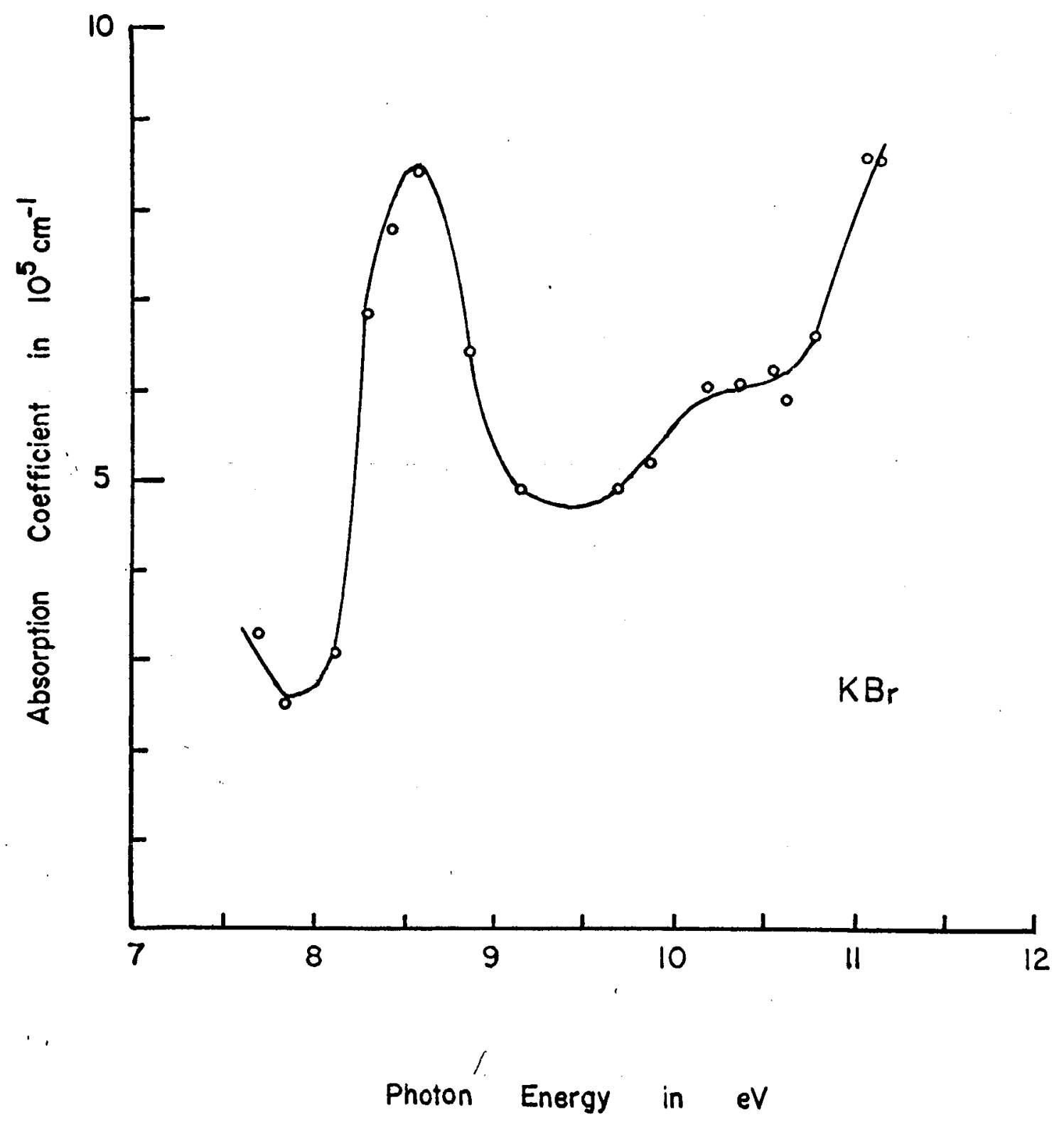




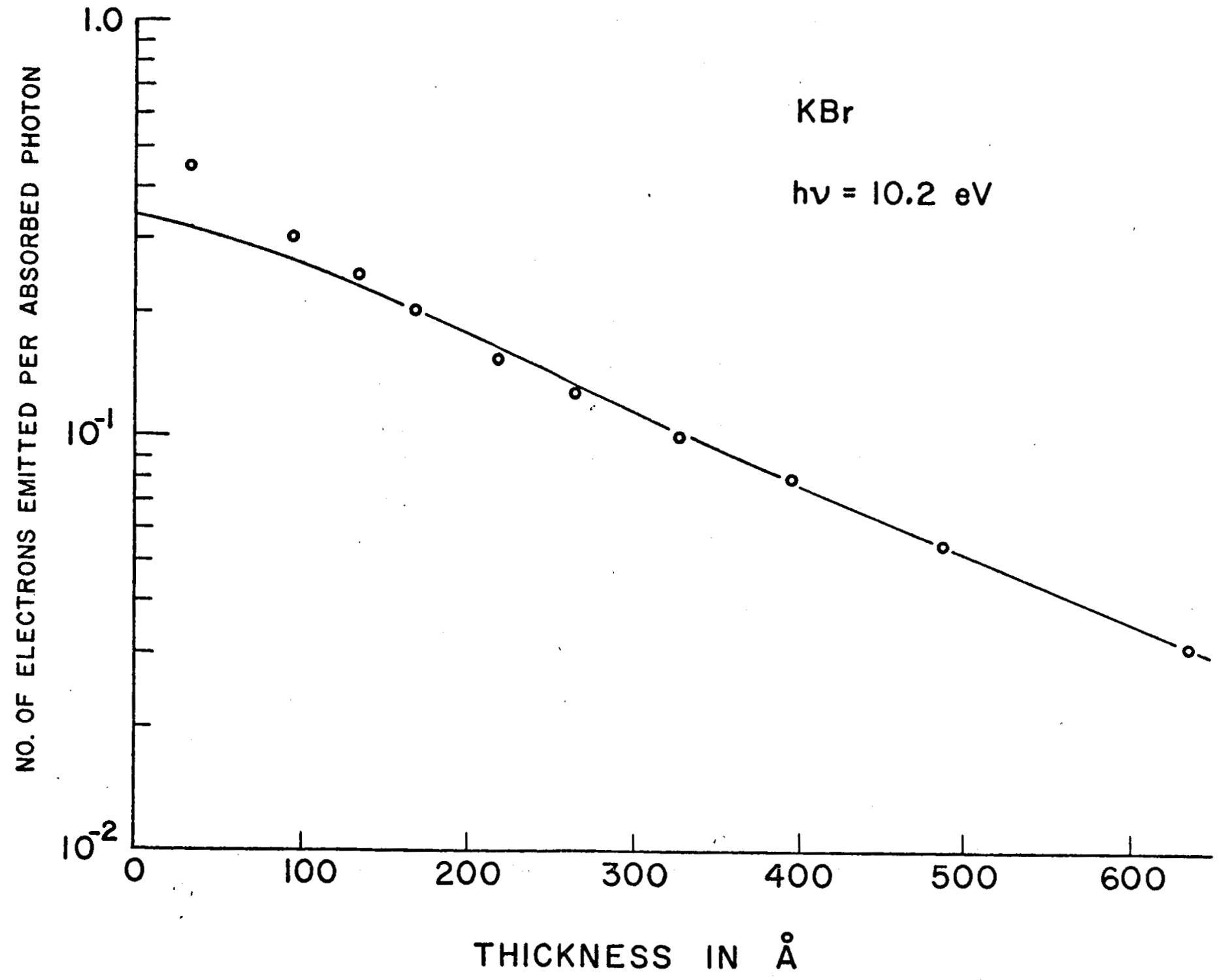




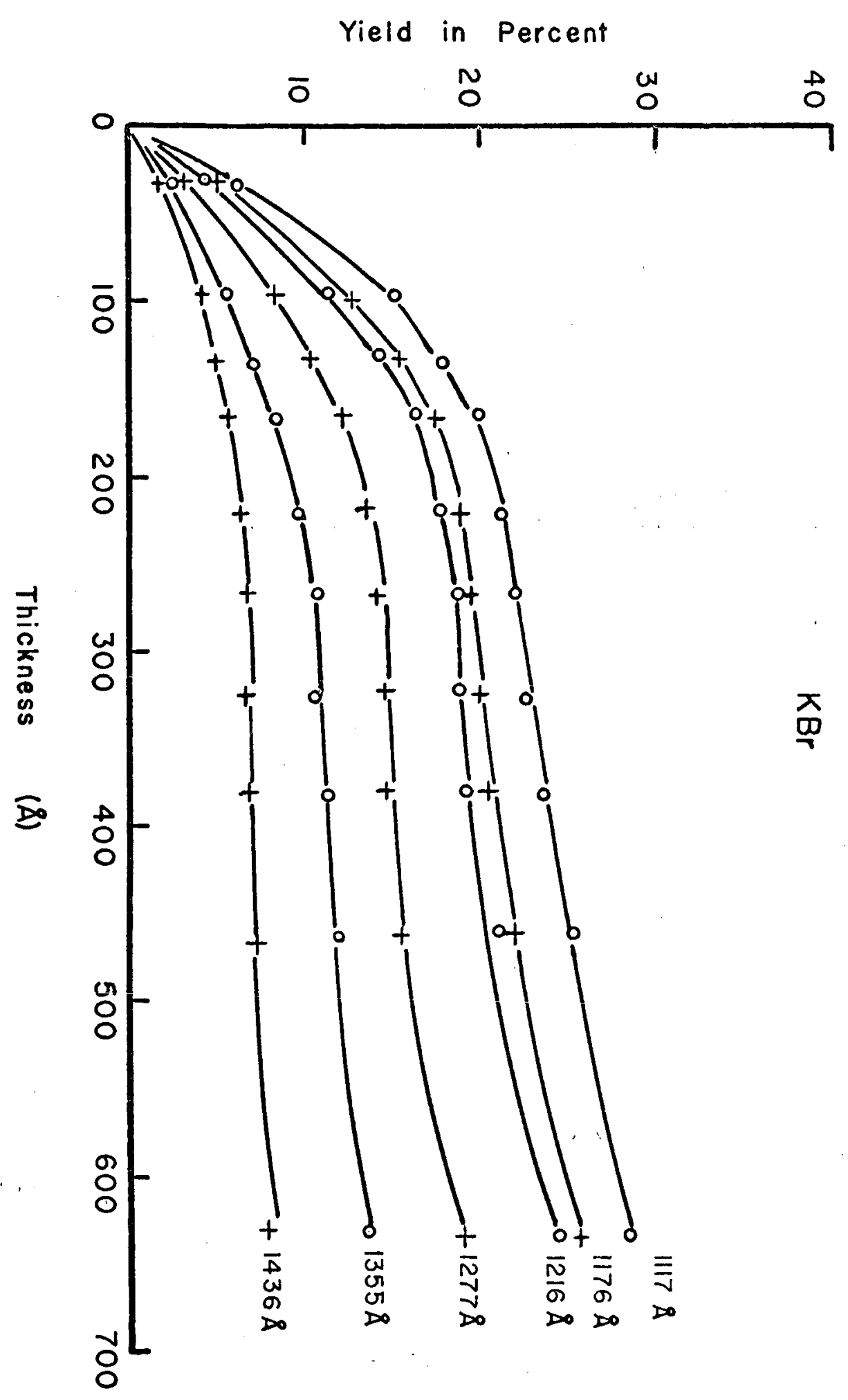

